\title{
Radiotherapy for urinary bladder pheochromocytoma with invasion of the prostate: A case report and literature review
}

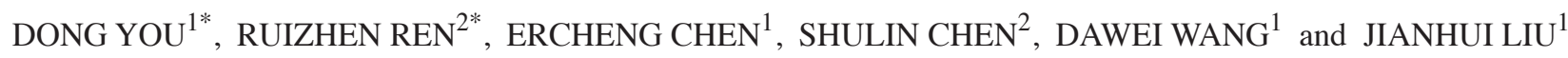 \\ Departments of ${ }^{1}$ Radiotherapy and ${ }^{2}$ Endocrinology, Yantai Yuhuangding Hospital \\ Affiliated to Qingdao University, Yantai, Shandong 264000, P.R. China
}

Received June 17, 2015; Accepted March 3, 2016

DOI: $10.3892 / \operatorname{mco} .2016 .820$

\begin{abstract}
Malignant pheochromocytoma is a rare tumor, for which there is currently no effective therapy. Cytoreductive surgery is recommended to reduce tumor burden and relieve the symptoms of catecholamine excess, although complete eradication of the lesions is often not feasible. In patients with advanced disease, for whom surgical resection is not an option, systemic chemotherapy, radiotherapy and treatment with iodine-131-meta-iodobenzylguanidine may be used to achieve symptomatic relief. Although malignant pheochromocytoma is considered to be unresponsive to radiotherapy, a limited number of case reports, although not large patient samples, have been published on the effectiveness of radiotherapy for the treatment of this disease. This is the case report of a 23 -year-old male patient with bladder pheochromocytoma invading the prostate, who refused to undergo surgery. The tumor shrank following radiotherapy and had not increased in size 1.5 years after treatment. Similarly, the blood pressure of the patient remained within normal limits without antihypertensive medication; the levels of catecholamines and their metabolites also remained normal. Our case demonstrated that radiotherapy was effective for malignant pheochromocytoma to a certain extent and, therefore, it may be selected when surgery is not feasible.
\end{abstract}

\section{Introduction}

Pheochromocytoma is a neoplasm of the chromaffin tissues, which mostly develops within the adrenal medulla. Only $\sim 15 \%$ of pheochromocytomas develop from extra-adrenal chromaffin tissues. Pheochromocytoma of the urinary bladder is rare, and

\footnotetext{
Correspondence to: Dr Ruizhen Ren, Department of Endocrinology, Yantai Yuhuangding Hospital Affiliated to Qingdao University, 20 Yuhuangding East Road, Yantai, Shandong 264000, P.R. China

E-mail: jennyrrz@sina.com

*Contributed equally
}

Key words: radiotherapy, bladder, pheochromocytoma, paraganglioma it originates from the chromaffin tissues of the sympathetic nervous system in the urinary bladder wall $(1,2)$. The typical signs are hematuria, hypertension during micturition, together with generalized symptoms due to the increased catecholamine levels, including headache, blurred vision, heart palpitations, profuse perspiration and flushing $(1,3)$. The standard treatment for localized or locally advanced pheochromocytomas is surgery, while metastatic or recurrent tumors are treated with palliative therapy (4-6).

We herein describe a case of primary urinary bladder pheochromocytoma with invasion of the prostate, without distant metastasis. The patient refused surgery and opted to receive radiotherapy.

\section{Case report}

A 23-year-old male patient presented with gross hematuria for 2 months, accompanied by hypertension for 20 days. The patient had no family history of hypertension and presented with no other symptoms. On admission, the blood pressure and pulse rate were $160 / 100 \mathrm{mmHg}$ and 76 beats/min, respectively. During micturition, the blood pressure increased to 185/110 $\mathrm{mmHg}$. The physical examination was otherwise unremarkable and the electrocardiography findings were normal.

Blood testing revealed high levels of norepinephrine (26.81 nmol/1; normal range, 0.31-2.82 nmol/1) and epinephrine (3.26 nmol/l; normal range, 0.03-0.98 nmol/l). A urinalysis revealed hematuria and elevated levels of vanillylmandelic acid (VMA) (121.9 $\mu \mathrm{mol} / 24 \mathrm{~h}$; normal range, $15.66-88.58 \mu \mathrm{mol} / 24 \mathrm{~h})$. The blood creatinine level was higher than normal $(165 \mu \mathrm{mol} / \mathrm{l}$; normal range, 71-115 $\mu \mathrm{mol} / 24 \mathrm{~h}$ ). Computed tomography (CT) revealed irregular thickening of the bladder wall, with an unclear boundary of the bladder and prostate gland. There was an effusion in the left renal pelvis, calyces and ureter. Magnetic resonance imaging (MRI) revealed a heterogeneous mass located in the urinary bladder and prostate (Fig. 1). The histopathological report confirmed the diagnosis of bladder pheochromocytoma with invasion of the prostate.

Although cystectomy and prostatectomy was considered to be the preferred treatment in this case, the patient refused surgery and opted to undergo radiotherapy; he received a total of 6,600 cGy, using 6-MV photon beams. The treatment was delivered in 30 fractions, $2.2 \mathrm{~Gy} /$ fraction, over 6 weeks. The 
patient completed the treatment course without severe complications or side effects.

An MRI conducted one year after radiotherapy revealed that the size of the tumor was clearly reduced (Fig. 2). The blood pressure remained within normal limits after discontinuing the antihypertensive medication, such as nifedipine controlled release tablets; serum norepinephrine, epinephrine and urinary VMA levels were also reduced and remained normal during the 1.5-year follow-up. However, the creatinine level increased significantly to $704 \mu \mathrm{mol} / 1$ shortly after radiotherapy. An ultrasound examination revealed that the left hydronephrosis was exacerbated and right hydronephrosis appeared following radiotherapy. A percutaneous nephrostomy drainage was performed and the creatinine decreased to nearly normal levels 1 month later; the drainage tube was then removed. A mild bilateral hydronephrosis persisted persisted for one year and the creatinine level was $120 \mu \mathrm{mol} / 11.5$ years later.

\section{Discussion}

Pheochromocytoma is a rare catecholamine-producing tumor, which commonly develops in the adrenal gland or paraaortic ganglia $(7,8)$. Approximately $3-13 \%$ of all pheochromocytomas are malignant, and extra-adrenal pheochromocytomas are more commonly malignant compared with intra-adrenal tumors. No reliable histological characteristics identify a pheochromocytoma as malignant, apart from the presence of distant metastases, such as to the locoregional lymph nodes. Metastasis is defined as appearance of chromaffin tissues at non-chromaffin sites distant from the primary tumor $(9,10)$. Pheochromocytoma of the bladder is rare and it arises from paraganglionic cells within the bladder wall; it often originates in the bladder trigone, and rarely in the anterior or lateral wall, which was the case in our patient $(1,2)$. The most common symptoms of bladder pheochromocytoma include hypertension, hematuria and other symptoms associated with raised catecholamine levels. However, certain patients may be asymptomatic $(1,3)$. Our patient only presented with hematuria and high blood pressure.

The biochemical diagnosis of pheochromocytoma is confirmed through measurement of the catecholamines or their metabolites in the plasma and urine (11). The blood norepinephrine and epinephrine and urinary VMA levels were increased in our patient, which supported the diagnosis of pheochromocytoma. The imaging methods used for diagnosis include ultrasonography, CT, MRI and meta-iodobenzylguanidine (MIBG) scintigraphy. CT may demonstrate the association between the mass and the surrounding tissues; however, it exhibits a high sensitivity for adrenal, but a lower sensitivity for extra-adrenal pheochromocytomas. MRI may also detect primary tumors and metastases, despite their small size, and it is also more sensitive compared with CT in the evaluation of the extra-adrenal pheochromocytomas (12-14). MIBG scintigraphy exhibits a high sensitivity and specificity and serves as a complementary diagnostic method $(15,16)$. Our patient was definitely diagnosed with pheochromocytoma by means of biochemical examinations, CT and MRI. Therefore, we did not consider a MIBG scan to be necessary. The histopathological report confirmed the diagnosis of bladder

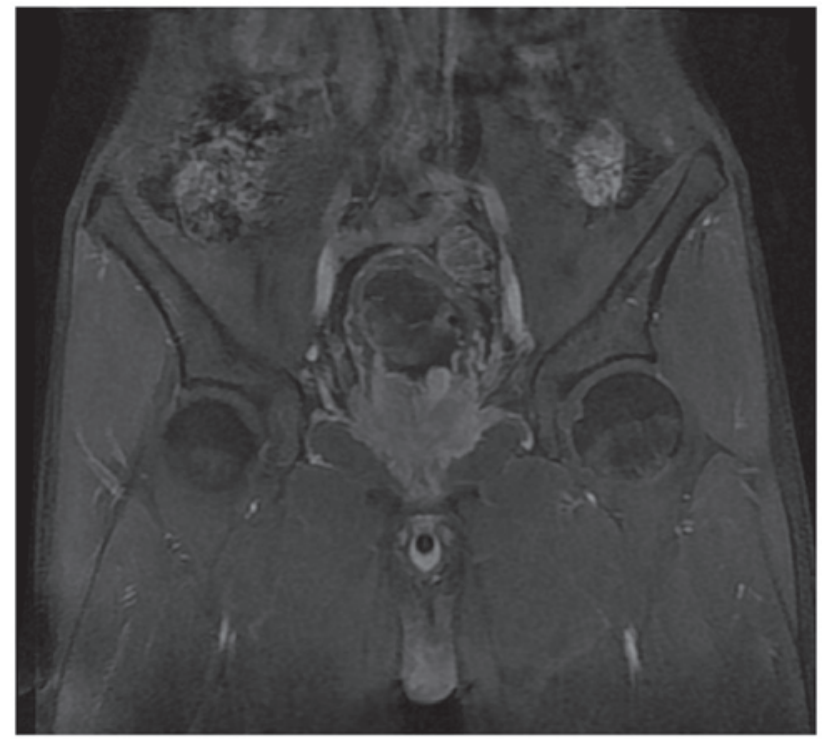

Figure 1. Magnetic resonance imaging (MRI) prior to radiotherapy. Enhanced MRI showing a lesion sized $60 \times 44 \times 63 \mathrm{~mm}$ in the prostate and bottom of the bladder, with an unclear boundary. The lesions exhibited marked inhomogeneous enhancement and the bladder wall thickening was not uniform.

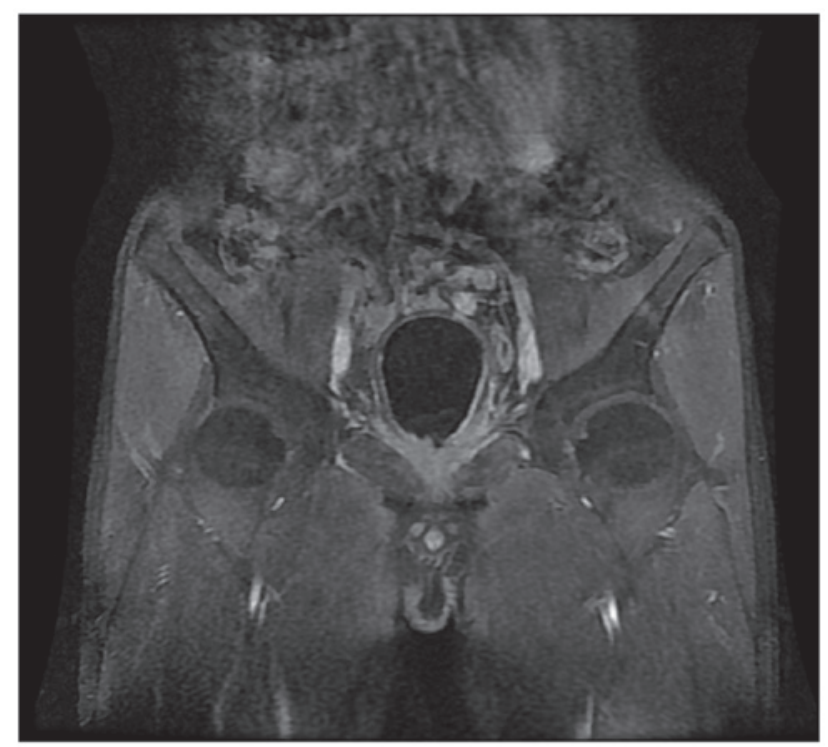

Figure 2. Magnetic resonance imaging (MRI) following radiotherapy. An enhanced MRI revealed that the size of the lesion in the prostate and bladder had decreased to $30 \times 25 \times 29 \mathrm{~mm}$ following radiotherapy. The boundary of the lesion was still unclear and the lesion exhibited inhomogeneous enhancement.

pheochromocytoma with invasion of the prostate, indicating that the tumor was malignant.

There is currently no effective treatment for malignant pheochromocytoma. Although complete eradication of the lesions may not be feasible, cytoreductive surgery is recommended to reduce tumor burden and the symptoms of catecholamine excess. In patients with advanced disease, for whom surgical resection is not an option, systemic chemotherapy, radiotherapy and treatment with ${ }^{131}$ I-MIBG may be used to achieve symptomatic relief (4-6). However, the evidence on the effectiveness of these treatments is limited for the majority of the 
cases. A limited number of case reports, although not large patient samples or controlled trials, have been published on the effectiveness of radiotherapy for malignant pheochromocytoma. Previous studies considered that radiotherapy was ineffective as a primary therapy and did not prevent local recurrence. However, radiotherapy achieved control of lymph node and bone metastases (17-19). Cystectomy and prostatectomy was considered to be the preferred treatment in the present case. However, the patient refused to undergo surgery. The tumor in the bladder trigone caused hydronephrosis and hydroureter, which resulted in postrenal renal failure. In order to relieve the hydroureter and hydronephrosis caused by the bladder pheochromocytoma, radiotherapy was selected. Our study demonstrated that radiotherapy led to tumor necrosis and caused the tumor to shrink gradually. Furthermore, the blood pressure was decreased and antihypertensive drugs were discontinued. In addition, the levels of catecholamines and their metabolites decreased to within the normal range.

Extra-adrenal pheochromocytomas are more likely to recur and metastasize; thus, long-term follow-up is required to detect any recurrence or metastasis $(20,21)$. Although the size of the lesion did not increase and the blood pressure remained normal 1.5 years later in the present case, lifelong follow-up is warranted.

The patient had left hydronephrosis prior to treatment and his creatinine level was marginally higher than the normal range. However, the left hydronephrosis was exacerbated and he developed right hydronephrosis shortly after radiotherapy. In addition, the creatinine level increased significantly. These changes may be attributed to the aggravation of the distal ureteral obstruction caused by edema of the ureteral orifice and the bladder trigone following radiotherapy. The hydronephrosis subsided and the creatinine level decreased following percutaneous nephrostomy drainage. The remission of hydronephrosis and decrease in creatinine levels following radiotherapy may be due to the relief of the ureteral obstruction caused by tumor shrinkage.

This case was reported due to the rarity of pheochromocytomas of the bladder with invasion of the prostate, and the current lack of effective treatment. The patient presented only with hematuria and high blood pressure, without any other typical symptoms of pheochromocytoma, despite increased levels of catecholamines and their metabolic products. Although malignant pheochromocytoma is considered to be resistant to radiation, and radiotherapy is not considered a primary treatment modality, in our case radiotherapy was effective for the treatment of malignant pheochromocytoma to a certain extent; therefore, radiotherapy may be selected when surgery is not feasible.

In conclusion, although malignant pheochromocytoma of the bladder is a rare occurrence, its diagnosis should be suspected in the presence of a bladder tumor accompanied by high blood pressure. There are currently no large-scale clinical studies comparing different therapeutic modalities in a randomized and controlled manner, due to the scarcity of cases with this type of tumor. Although malignant pheochromocytoma is generally considered to be unresponsive to radiotherapy, our case demonstrated that radiotherapy was effective to a certain extent and, therefore it may be selected when surgery is not an option.

\section{References}

1. Doran F, Varinli S, Bayazit Y, Bal N and Ozdemir S: Pheochromocytoma of the urinary bladder. APMIS 110: 733-736, 2002.

2. Onishi T, Sakata Y, Yonemura $S$ and Sugimura $Y$ : Pheochromocytoma of the urinary bladder without typical symptoms. Int J Urol 10: 398-400, 2003.

3. Naqiyah I, Rohaizak M, Meah FA, Nazri MJ, Sundram M and Amram AR: Phaeochromocytoma of the urinary bladder. Singapore Med J 46: 344-346, 2005.

4. Nazario J and Gupta S: Transarterial liver-directed therapies of neuroendocrine hepatic metastases. Semin Oncol 37: 118-126, 2010.

5. Pacak K, Eisenhofer G, Ahlman H, Bornstein SR, Gimenez-Roqueplo AP, Grossman AB, Kimura N, Mannelli M, McNicol AM and Tischler AS; International Symposium on Pheochromocytoma: Pheochromocytoma: Recommendations for clinical practice from the first international symposium. October 2005. Nat Clin Pract Endocrinol Metab 3: 92-102, 2007.

6. Vogl TJ, Naguib NN, Zangos S, Eichler K, Hedayati A and Nour-Eldin NE: Liver metastases of neuroendocrine carcinomas: Interventional treatment via transarterial embolization, chemoembolization and thermal ablation. Eur J Radiol 72: 517-528, 2009.

7. Bravo EL and Tagle R: Pheochromocytoma: State-of-the-art and future prospects. Endocr Rev 24: 539-553, 2003.

8. Neumann HP, Bausch B, McWhinney SR, Bender BU, Gimm O, Franke G, Schipper J, Klisch J, Altehoefer C, Zerres K, et al: Germ-line mutations in nonsyndromic pheochromocytoma. N Engl J Med 346: 1459-1466, 2002.

9. Eisenhofer G, Bornstein SR, Brouwers FM, Cheung NK, Dahia PL, de Krijger RR, Giordano TJ, Greene LA, Goldstein DS, Lehnert $\mathrm{H}$, et al: Malignant pheochromocytoma: Current status and initiatives for future progress. Endocr Relat Cancer 11: 423-436, 2004

10. Scholz T, Schulz C, Klose S and Lehnert H: Diagnostic management of benign and malignant pheochromocytoma. Exp Clin Endocrinol Diabetes 115: 155-159, 2007.

11. Kvetnansky R, Sabban EL and Palkovits M: Catecholaminergic systems in stress: Structural and molecular genetic approaches. Physiol Rev 89: 535-606, 2009.

12. Vyas S, Kalra N, Singh SK, Agarwal MM, Mandal AK and Khandelwal N: Pheochromocytoma of urinary bladder. Indian J Nephrol 21: 198-200, 2011.

13. Wang H, Ye H, Guo A, Wei Z, Zhang X, Zhong Y, Fan Z, Wang Y and Wang D: Bladder paraganglioma in adults: MR appearance in four patients. Eur J Radiol 80: e217-e220, 2011.

14. Whalen RK, Althausen AF and Daniels GH: Extra-adrenal pheochromocytoma. J Urol 147: 1-10, 1992.

15. Berglund AS, Hulthén UL, Manhem P, Thorsson O, Wollmer P and Törnquist C: Metaiodobenzylguanidine (MIBG) scintigraphy and computed tomography $(\mathrm{CT})$ in clinical practice. Primary and secondary evaluation for localization of phaeochromocytomas. J Intern Med 249: 247-251, 2001.

16. Nakatani T, Hayama T, Uchida J, Nakamura K, Takemoto Y and Sugimura K: Diagnostic localization of extra-adrenal pheochromocytoma: Comparison of ${ }^{123}$ I-MIBG imaging and ${ }^{131}$ I-MIBG imaging. Oncol Rep 9: 1225-1227, 2002.

17. Naguib M, Caceres M, Thomas CR Jr, Herman TS and Eng TY: Radiation treatment of recurrent pheochromocytoma of the bladder: Case report and review of literature. Am J Clin Oncol 25: 42-44, 2002

18. Tato A, Orte L, Diz P, Quereda C and Ortuno J: Malignant pheochromocytoma, still a therapeutic challenge. Am J Hypertens 10: 479-481, 1997.

19. Yu L, Fleckman AM, Chadha M, Sacks E, Levetan C and Vikram B: Radiation therapy of metastatic pheochromocytoma: Case report and review of the literature. Am J Clin Oncol 19: 389-393, 1996.

20. Guller U, Turek J, Eubanks S, Delong ER, Oertli D and Feldman JM: Detecting pheochromocytoma: Defining the most sensitive test. Ann Surg 243: 102-107, 2006.

21. Zeitlin I, Dessau H, Lorberboym M and Beigel Y: Malignant pheochromocytoma of the urinary bladder: Challenges in diagnosis and management. Isr Med Assoc J 13: 311-313, 2011. 